NBSIR 77-1283

\title{
Spectra From Energy Degradation of Fast Electrons in Water
}

Martin J. Berger

Center for Radiation Research Institute for Basic Standards National Bureau of Standards Washington, D.C. 20234

\section{August 1977}

Interim Report for Period October 1, 1976 to July 15, 1977

\section{Prepared for}

Office of Naval Research

Arlington, Virginia 22217

and

Energy Research and Development Administration Washington, D.C. 20545 



\section{SPECTRA FROM ENERGY}

DEGRADATION OF FAST ELECTRONS

IN WATER

Martin J. Berger

Center for Radiation Research Institute for Basic Standards National Bureau of Standards

Washington, D.C. 20234

August 1977

Interim Report for Period October 1, 1976 to July 15, 1977

Prepared for

Office of Naval Research

Arlington, Virginia 22217

and

Energy Research and Development Administration

Washington, D.C. 20545

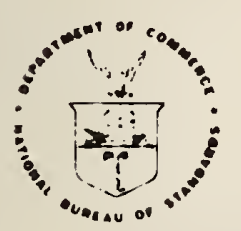

U.S. DEPARTMENT OF COMMERCE, Juanita M. Kreps, Secretary

Dr. Sidney Harman, Under Secretary

Jordan J. Baruch. Assistant Secretary for Science and Technology

NATIONAL BUREAU OF STANDARDS, Ernest Ambler, Acting Director 

SPECTRA FROM ENERGY DEGRADATION OF FAST ELECTRONS IN WATER ${ }^{*}$

Martin J. Berger

\begin{abstract}
This report gives data for energy degradation spectra in water vapor. Calculations utilized the Monte Carlo method and included Auger electrons. Results include 1) a new energy scaling principle at low energies, 2) values at different source energies for $W$, the mean energy loss per ion pair formed, 3) data on the electron range calculated by continuous-slowing-down approximation, and 4) data on stopping powers and restricted stopping powers as compared with results from the Bethe theory.
\end{abstract}

\footnotetext{
* Work supported by Office of Naval Research, Arlington, Va. and U.S. Energy Research and Development Admin., Washington, D.C. $+$

This is an interim report and the work is continuing. The report is to be superseded by a future publication which will receive general distribution and should be cited as a reference. Please consult the NBS office of Technical Publications to obtain the proper citation.
} 



\section{INTRODUCTION}

Fast electrons lose energy to the surrounding medium by many $k$ inds of interactions, including a) knock-on processes with individual electrons which follow approximately the Mфller cross section, and b) glancing processes for which the electric pulse generated when a fast electron passes an atom can stimulate both excitation and ionization.

The cumulative result of these interactions is the progressive loss of energy, often called energy "degradation," by the fast electrons. A source of electrons can thus give rise to electrons of all lower energies; and these electrons include not only the "primaries" whose energies have been degraded, but also "secondaries" produced in ionizing interactions and defined to be the free electron of lower energy of the two which emerge from such interactions. The resulting spectrum is commonly called the "degradation" spectrum; and it has importance in many fields dealing with radiation effects on matter.

Since the early exploratory calculations of Spencer and Fano (1954), calculations of these spectra for a variety of materials have been made; although the requirement for a complete set of inelastic cross sections at all energies below the source energy $\mathrm{T}_{0}$ makes these calculations difficult. In this brief interim report we give many of our results on degradation spectra for water vapor. We have not yet completed comparisons with other calculations, including those for comparable materials; these will be included in the final publication on this subject. 

The Monte Carlo method has been applied to the calculation of energy degradation spectra in water vapor. Calculations have been made at a large number of energies between $100 \mathrm{keV}$ and a few eV. The successive inelastic events which an electron makes in the course of slowing down in an extended medium have been simulated in direct analogy to the physical processes, and all generations of secondary electrons were followed. The number of electrons whose energy histories were simulated varied depending on the initial energy $\mathrm{T}_{\mathrm{o}}$ of the primary electron; for example:

$\underline{T_{0}, e V}$

100,000

10,000

1,000

100
No. of primaries followed

500

2,000

10,000
No. of secondaries followed

per primary, on the average

3270

331

33

As far as possible, the various inelastic events were sampled according to cross sections derived from experiments. The sources of cross section data were as follows:

Excitation.................... Olivero, Stagat and Green (1972); Kutcher and Green (1976)

Dissociative excitation............. Beenakker, de Heer, Krop and Möhlmann (1974)

Dissociative attachment........... Compton and Christophorou (1967)

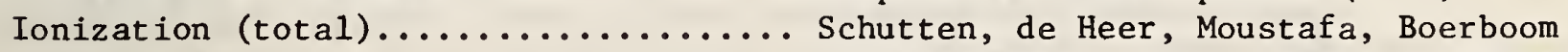
and Kistemaker (1966); Maerk and Egger (1975)

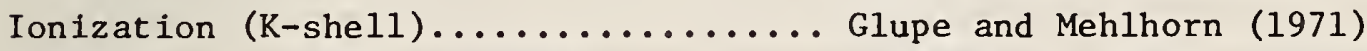

Spectrum of Auger electrons............Moddeman, Carlson, Krause and Pullen (1971)

Ionization (differential)........... Opal and Beaty (1972) 
Altogether 27 possible modes of excitation were taken into consideration, and use was made of the very convenient parameterization of the excitation cross sections by Green and collaborators. The Opal-Beaty ionization cross section, differential in the secondary electron energy, was available only at $500 \mathrm{eV}$. It was fitted at that energy by a simple analytical formula which is a modification of the Mфller cross section (for scattering by free electrons) and which takes into account exchange effects). Following Kim (1975) it was assumed that the spectrum of energy transfers in glancing collisions is determined predominantly by the oscillator-strength distribution, and is nearly the same at all energies. The simple analytical formula was therefore retained, but was renormalized at each energy to obtain agreement with the experimental total ionization cross section. At spectral energies from 200 to $600 \mathrm{eV}$ the ionization cross section was made to go over into the Mфller cross section. Ionization was assumed to be possible from five orbitals, with binding energies of $12.6,14.7,18.4,32.2$ and $540 \mathrm{eV}$. The relative probability of electron ejection from the K-shell (followed by emission of an Auger electron) was estimated from experimental data; the relative probabilities of ejection from the other orbitals was estimated from a Weizsacker-Williams calculation by Seltzer (private communication).

\section{RESULTS}

We summarize here certain significant conclusions that can be drawn from the analysis of results performed so far, and back them up with information in tabular form.

1. As shown in Table 1, the energy degradation spectra calculated with the use of detailed inelastic collision cross sections are in good agreement at high and intermediate energies with the results of the Spencer-Fano (1954) theory. The latter assumes energy transfers to be governed by the M $\phi 11$ er cross section, adjusted with a cut-off on low-energy transfers to give the correct stopping power. It turns out that this approximation gives good results for spectral energies down to $200 \mathrm{eV}$; below this energy the SpencerFano theory is no longer applicable. This presumably holds not only for water vapor but also for other gases of similar atomic number. Below $200 \mathrm{eV}$, the new Monte Carlo results are the appropriate extension of the Spencer-Fano theory into the low-energy region of interest for radiation chemistry yield studies.

2. A new and useful scaling law has been discovered, which applies at low spectral energies, say, below a few hundred eV, and which greatly reduces, and at low energies practically suppresses the dependence of the energy degradation spectra on the initial electron energy. It has been customary to represent the energy degradation spectrum in terms of the differential tracklength distribution $\mathrm{y}\left(\mathrm{T}, \mathrm{T}_{0}\right)$, defined such that $\mathrm{y}(\mathrm{T}, \mathrm{T}) \mathrm{dT}$ is the average tracklength of electrons with energies between $\mathrm{T}^{\mathrm{O}}$ and $\mathrm{T}+\mathrm{dT}$. The new scaled quantity is

$$
\mathrm{T} \frac{\mathrm{dG}}{\mathrm{dT}}=\mathrm{T}\left(100 / \mathrm{T}_{\mathrm{o}}\right)(\mathrm{N} \sigma / \rho) \mathrm{y}\left(\mathrm{T}, \mathrm{T}_{\mathrm{o}}\right)
$$


Here $T$ is the spectral energy and $T$ the source energy, both in $\mathrm{eV} ; \mathrm{N}$ is the number of molecules per $\mathrm{cm}_{2}$, $\sigma$ is the total cross section for inelastic collisions, in $\mathrm{cm}^{2}$, and $\rho$ is the density of the medium, in $\mathrm{g} / \mathrm{cm} ; \mathrm{y}\left(\mathrm{T}, \mathrm{T}_{\mathrm{m}}\right)$ is the differential tracklength distribution in $\mathrm{g} / \mathrm{cm}^{2}$ per $\mathrm{e} \theta$. The quantity $\mathrm{dG} / \mathrm{dT}$ can be interpreted as the differential yield of inelastic collisions per unit spectral energy, and is normalized to an energy input of $100 \mathrm{eV}$. The dimensionless quantity $\mathrm{T} d \mathrm{~d} / \mathrm{dT}$ is given in Table 2 for initial electron energies $\mathrm{T}$ between 100,000 and $1000 \mathrm{eV}$. It can be seen that, as claimed, the dependence of $\mathrm{T} d \mathrm{~d} / \mathrm{dT}$ on $\mathrm{T}$ becomes progressively weaker as the spectral energy $\mathrm{T}$ decreases. $T$ This suggests that energy degradation spectra for values of $T$ much higher than $100 \mathrm{keV}$ could be obtained without much trouble by ${ }^{\circ}$ using the Spencer-Fano theory down to $200 \mathrm{eV}$, and adding on the low-energy part of the spectrum assuming a universal spectral shape. Finally, we note that the yield $G_{i}\left(T_{0}\right)$ of events of type $i$ can be calculated from the expression

$$
G_{i}\left(T_{0}\right)=\int_{0}^{T_{0}}(d G / d T)\left(\sigma_{i} / \sigma\right) d T,
$$

where $\sigma_{i}$ is the cross section for the event of type $i$. Again, the yield is normalized to an energy input of $100 \mathrm{eV}$.

3. Auger electrons emitted after ionization from the K-shell make a significant contribution to the energy degradation spectrum at spectral energies below $500 \mathrm{eV}$. The magnitude of this contribution is given in Table 3. Many previous treatments of the energy degradation spectrum, including that of spencer and Fano, have omitted the Auger electron contribution. Therefore the comparison given in Table 2 does not include Auger electrons.

4. In the course of the Monte Carlo calculation of the energy degradation spectra, the yields of various events have also been obtained, for example, the yield of ionizations. Table 4 gives the mean number of ionizations as a function of the initial electron energy, as well as the ionization fluctuations as summarized in terms of the Fano factor, which is the ratio of the mean square to the mean number of ionizations. If the fluctuations were governed by a Poisson distribution, the Fano factor would be unity. As can be seen from Table 4, this is the case only quite close to the ionization threshold $(12.6 \mathrm{eV})$. At higher energies the Fano factor assumed a limiting value of 0.25 .

5. The value of $W$ (mean energy needed to produce an ion pair in water vapor) is shown in Table 5. It can be seen that $W$ is almost constant down to energies of a few hundred $\mathrm{eV}$, whereupon it rises at first slowly and then quite rapidly at lower energies. Between $10 \mathrm{keV}$ and $100 \mathrm{keV}$ there is a very slow rise of $1.1 \%$. It would not be altogether surprising if this rise continued as the electron 
energy is further increased. Table 6 compares our value of $W$ with that obtained in other calculations, with differences presumably arising mainly from the different assumed cross sections, but to some extent also from the calculational techniques. The experimental W-value for water vapor is given as $29.9 \mathrm{eV}$ by Adler and Bother (1965) and as $30.1 \mathrm{eV}$ by Booz and Ebert (1963). These values differ by $1.3 \%$ and $0.7 \%$, respectively, from the result at $\mathrm{T}_{0}=1 \mathrm{keV}$ of the present calculation, differences which are well within the combined limits of experimental and theoretical uncertainties.

6. The contribution of ionization events from different spectral regions of the energy degradation spectrum is indicated in Table 7. It can be seen that a significant contribution to the ionization is made by high-energy as well as by low-energy electrons. Moreover, the relative contributions from different spectral regions are seen to be rather insensitive to the initial energy of the primary electron, if scaling by $\mathrm{T}_{0}$ is used for the top half of the table.

7. From the inelastic scattering cross sections compiled for the Monte Carlo calculation, one can also derive the electron stopping power. Table 8 compares the results thus obtained with the values given by the Bethe stopping-power formula (assuming a mean excitation energy of $71.7 \mathrm{eV}$ for water vapor). Between $100 \mathrm{keV}$ and $20 \mathrm{keV}$, there is no difference. At lower energies, the present results fall below the results from the Bethe theory, by $1.3 \%$ at $10 \mathrm{keV}, 6.8 \%$ at $5 \mathrm{keV}, 8.3 \%$ at $2 \mathrm{keV}$ and $11 \%$ at $1 \mathrm{keV}$.

8. The CSDA (continuous-slowing-down-approximation) range has been computed from the new stopping-power values in Table 7 , and is given in Table 9. The range is computed as the pathlength which the electron would travel until it reaches a cut-off energy $\mathrm{T}$, assuming that the energy loss along the entire path is always equal to the mean loss, i.e., the stopping-power. The results in Table 9 are for a cut-off $\mathrm{T}_{\mathrm{c}}=12.6 \mathrm{eV}$, equal to the lowest ionization potential in water vapor. Depending on the chosen value of $\mathrm{T}_{c}$, different range values are obtained; for example:

CSDA range, $\mathrm{g}^{-2}$

\begin{tabular}{rlll}
$\mathrm{T}_{\mathrm{o}}, \mathrm{keV}$ & $\mathrm{T}_{\mathrm{c}}=4.5 \mathrm{eV}$ & $\mathrm{T}_{\mathrm{c}}=12.6 \mathrm{eV}$ & $\mathrm{T}_{\mathrm{c}}=20.0 \mathrm{eV}$ \\
\hline 100 & $1.522 \times 10^{-2}$ & $1.432 \times 10^{-2}$ & $1.423 \times 10^{-2}$ \\
10 & $1.246 \times 10^{-2}$ & $2.828 \times 10^{-4}$ & $2.599 \times 10^{-4}$ \\
1 & $0.926 \times 10^{-4}$ & $7.156 \times 10^{-6}$ & $6.500 \times 10^{-6}$
\end{tabular}

9. In radiological physics, it is often of interest ot know the "local" energy deposition in terms of restricted stopping power, i.e., a mean energy loss per unit pathlength from events in which the energy transfer from the electron to the molecule is smaller than some chosen cut-off energy $\Delta$. In cavity ionization theory, the $\Delta$ is typically 
10 to $15 \mathrm{keV}$, and the Bethe theory for the restricted stopping power can be applied above $\sim 10 \mathrm{keV}$ in water. In track structure theory in radiobiology, cut-off energies $\Delta$ as $10 \mathrm{w}$ as $100 \mathrm{eV}$ are considered. As is shown in Table 10, the cross sections assumed in the present work then lead to significant departures from the predictions of the Bethe theory, even for electron energies as high as $100 \mathrm{keV}$.

\section{REFERENCES}

Adler, P. and Bothe, H. K., (1965), Zeit. Naturforsch 20a, 1700.

Beenakker, C.I.M., De Heer, F. J., Krop, H. B., and Möhlmann, G. R., (1974) Chem. Phys. 6, 445.

Compton, R.N., and Christophorou, L.G., (1967), Phys. Rev. 154, 110.

Booz, J. and Ebert, H.G. (1963), Strahlentherapie, 120, 7.

Glupe, G. and Mehlhorn, W. (1971), J. Phys. 4, 40.

Kim, Y. K. (1975), Radiat. Res. 61, 21; 64, 205.

Kutcher, G. J. and Green, A.E.S., (1976), Radiat. Res. 67, 408.

Moddeman, W.E., Carlson, T.A., Krause, M.O., and Pullen, B.P. (1971), J. Chem. Phys. 55, 2317.

Olivero, J.J., Stagat, R.W., and Green, A.E.S., (1972), J. Geophys. Res. 77, 4797

Opal, C.B., Beaty, E.C., and Peterson, W. K., (1972) Atomic Data 4, 209

Paretzke, H. W., (1976), Proc. 5th Symposium on Microdosimetry, Euratom Publication $5452 \mathrm{~d}-\mathrm{e}-\mathrm{f}$.

Schutten, J., De Heer, A.J., Moustafa, H.R., Boerboom, A.J.H., and Kistemaker, J. (1966), J. Chem. Phys. 44, 3924.

Spencer, L.V and Fano, U., (1954), Phys. Rev. 93, 1172. 



$$
\left[y\left(T, T_{0}\right) S(T)\right] /\left[y\left(T, T_{0}\right) F\left(T, T_{0}\right)\right]_{\text {Spencer-Fano }}
$$

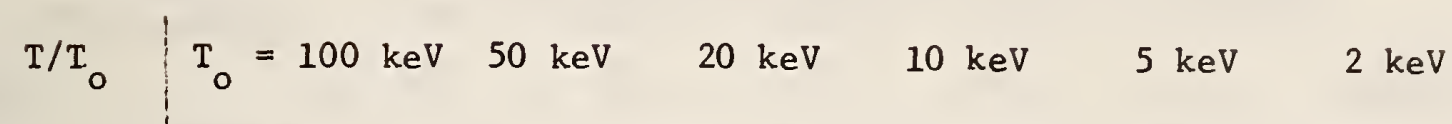

\begin{tabular}{l|llllll}
\hline $1 / 2^{-1 / 6}$ & 1.048 & 1.059 & 1.088 & 1.098 & 1.075 & 1.117 \\
$1 / 2^{-2 / 6}$ & 0.988 & 0.996 & 1.012 & 1.003 & 0.983 & 1.012 \\
$1 / 2^{-3 / 6}$ & 0.966 & 0.992 & 0.989 & 0.983 & 0.979 & 0.986 \\
$1 / 2^{-4 / 6}$ & 0.963 & 1.000 & 0.988 & 0.989 & 0.982 & 0.968 \\
$1 / 2^{-5 / 6}$ & 0.972 & 1.005 & 0.994 & 0.997 & 0.971 & 0.966 \\
$1 / 2$ & 0.983 & 0.993 & 0.996 & 0.997 & 0.962 & 0.970 \\
$1 / 4$ & 1.013 & 1.013 & 0.985 & 0.986 & 0.997 & 0.981 \\
$1 / 8$ & 0.991 & 0.998 & 0.999 & 0.991 & 0.995 & 0.953 \\
$1 / 16$ & 1.001 & 0.970 & 0.983 & 0.997 & 1.008 & \\
$1 / 32$ & 0.964 & 0.966 & 0.997 & 1.010 & 0.890 & \\
$1 / 64$ & 0.970 & 0.972 & 1.031 & 0.921 & & \\
$1 / 128$ & 0.964 & 1.001 & & & & \\
$1 / 256$ & 0.997 & 1.050 & & & & \\
$1 / 512$ & 1.068 & & & & & \\
\hline
\end{tabular}

Comparison of energy-degradation spectra from the present work and the corresponding spectra from the Spencer-Fano theory. $\mathrm{T}_{0}$ is the source energy, $T$ the spectral energy, $y\left(T, T_{0}\right)$ the differential tracklength distribution, $S(T)$ the electron stopping power, and $F\left(T, T_{0}\right)$ the quantity which plays the role of a stopping power in the Spencer-Fano theory. 
$\mathrm{T} d G\left(T, T_{0}\right) / d T$

\begin{tabular}{rlllllll}
\multicolumn{1}{c}{$\mathrm{T}_{\mathrm{eV}} \mathrm{T}_{\mathrm{o}}=100,000$} & 50,000 & 20,000 & 10,000 & 5000 & 2000 & $1000 \mathrm{eV}$ \\
50,000 & 1.03 & & & & & & \\
20,000 & 0.462 & 0.822 & & & & & \\
10,000 & 0.274 & 0.475 & 1.08 & & & & \\
5000 & 0.217 & 0.300 & 0.589 & 1.14 & & & \\
2000 & 0.200 & 0.230 & 0.345 & 0.552 & 1.00 & & \\
1000 & 0.222 & 0.243 & 0.309 & 0.405 & 0.613 & 1.35 & \\
500 & 0.272 & 0.286 & 0.327 & 0.376 & 0.485 & 0.838 & 1.52 \\
200 & 0.513 & 0.509 & 0.529 & 0.522 & 0.550 & 0.653 & 0.882 \\
100 & 0.684 & 0.692 & 0.691 & 0.697 & 0.707 & 0.740 & 0.832 \\
50 & 1.00 & 1.00 & 1.01 & 1.01 & 1.02 & 1.03 & 1.06 \\
20 & 1.60 & 1.61 & 1.61 & 1.62 & 1.64 & 1.65 & 1.66 \\
10 & 1.88 & 1.88 & 1.89 & 1.90 & 1.89 & 1.91 & 1.92 \\
5 & 1.73 & 1.73 & 1.74 & 1.75 & 1.77 & 1.78 & 1.80 \\
& & & & & & & \\
\hline
\end{tabular}

Scaled energy degradation spectra in water vapor. The quantity dG/dT is the differential yield, per spectral energy interval, of inelastic collisions produced by the primary electrons and all their secondaries. The differential yield is normalized to an energy input of $100 \mathrm{eV}$ into the medium. 
TABLE 3

ENERGY

INTERVAL

\begin{tabular}{rrrrrrr}
$(\mathrm{eV})$ & $\mathrm{T}_{\mathrm{o}}=100$ & 50 & 20 & 10 & 5 & 2 \\
\cline { 2 - 6 } $500-490$ & 55.0 & 50.8 & 44.1 & 35.4 & 25.1 & 8.4 \\
$490-480$ & 39.3 & 35.3 & 30.4 & 22.4 & 12.7 & 4.6 \\
$400-380$ & 27.9 & 26.0 & 22.4 & 15.6 & 11.5 & 3.4 \\
$300-280$ & 20.9 & 19.2 & 16.1 & 11.1 & 8.9 & 3.3 \\
$200-190$ & 13.3 & 12.3 & 11.3 & 8.1 & 5.4 & 2.4 \\
$100-96$ & 7.1 & 6.5 & 6.1 & 5.2 & 3.6 & 2.0 \\
$50-48$ & 5.7 & 5.1 & 4.4 & 3.0 & 2.4 & 1.3 \\
$30-28$ & 5.0 & 4.6 & 4.0 & 2.8 & 2.3 & 1.0 \\
$20-19$ & 4.9 & 4.4 & 3.9 & 3.0 & 2.4 & 1.1 \\
$10-9.6$ & 4.9 & 4.4 & 4.0 & 2.8 & 2.4 & 0.8 \\
$5-4.8$ & 4.9 & 4.4 & 3.8 & 2.6 & 2.4 & 1.1
\end{tabular}

Percent contribution by Auger electrons (and their secondaries) to the energy degradation spectrum. The contributions shown are averaged over the indicated energy intervals. 
TABLE 4

\begin{tabular}{rcc}
$T_{0}$ & $\overline{n_{i}}$ & $\overline{n_{i}^{2}} / \overline{n_{i}}$ \\
\hline 12.6 & 0.0 & 1.00 \\
13 & 0.037 & 0.96 \\
14 & 0.139 & 0.86 \\
15 & 0.213 & 0.79 \\
20 & 0.347 & 0.65 \\
50 & 1.225 & 0.40 \\
100 & 2.822 & 0.33 \\
200 & 6.152 & 0.28 \\
500 & 16.24 & 0.25 \\
1000 & 32.98 & 0.24 \\
2000 & 66.33 & 0.24 \\
5000 & 165.9 & 0.24 \\
10,000 & 330.7 & 0.25 \\
20,000 & 659.1 & 0.25 \\
50,000 & 1639. & 0.25 \\
100,000 & 3270. & 0.25 \\
\hline
\end{tabular}

Mean number of ions produced, $\overline{n_{i}}$, and Fano factor $f=\overline{n_{i}^{2}} / \overline{n_{i}}$, for electroas of initial energy $T_{0}$ slowed down in water vapor. 


\begin{tabular}{rl}
$\begin{array}{c}\mathrm{T}_{\mathrm{O}} \\
(\mathrm{eV})\end{array}$ & $\mathrm{W}$ \\
\hline 20 & 57.67 \\
30 & 47.07 \\
50 & 40.81 \\
100 & 35.43 \\
200 & 32.51 \\
300 & 31.54 \\
500 & 30.78 \\
1000 & 30.32 \\
2000 & 30.15 \\
3000 & 30.17 \\
5000 & 30.14 \\
10,000 & $30.23_{5}$ \\
20,000 & $30.34_{5}$ \\
30,000 & $30.44_{5}$ \\
50,000 & $30.51_{5}$ \\
100,000 & 30.58 \\
\hline
\end{tabular}

Average energy needed for the production of an ion pair, $W$, for electrons with initial energy $T_{0}$ in water vapor. 


\begin{tabular}{|c|c|c|c|c|}
\hline$(\mathrm{eV})$ & OSG & KG & $\mathrm{P}$ & $\begin{array}{l}\text { Present } \\
\text { Work }\end{array}$ \\
\hline 20 & $7 \overline{73.6}$ & 82.5 & 44.6 & 57.7 \\
\hline 40 & 33.3 & 43.2 & 36.9 & 43.2 \\
\hline 60 & 26.9 & 37.9 & 34.6 & 39.0 \\
\hline 80 & 25.4 & 35.8 & 33.4 & 37.0 \\
\hline 100 & 25.0 & 34.9 & 32.7 & 35.4 \\
\hline 200 & 25.0 & 33.2 & 31.2 & 32.5 \\
\hline 400 & 25.0 & 31.7 & 30.6 & 31.0 \\
\hline 600 & 25.0 & 30.9 & 30.5 & 30.6 \\
\hline 800 & 25.0 & 30.6 & 30.4 & 30.4 \\
\hline 1000 & 25.0 & 30.0 & 30.4 & 30.3 \\
\hline
\end{tabular}

Value of $W$, the mean energy needed to produce an ion pair, as calculated by various authors.

OSG: Olivero, Stagat and Green (1972)

KG: Kutcher and Green (1976)

$\dot{\mathrm{P}}$ : Paretzke (1975)

The results of Kutcher and Green are for 1iquid water; all the others are for water vapor. 
TABLE 7

\begin{tabular}{rrrl} 
& \multicolumn{3}{c}{ Spectral energy $\mathrm{T}, \mathrm{keV}$} \\
\cline { 2 - 4 } 100 & 100.0 & 10.0 & 1.0 \\
90 & 81.1 & 8.41 & 0.921 \\
80 & 56.6 & 6.12 & 0.765 \\
70 & 29.5 & 3.96 & 0.588 \\
60 & 7.43 & 1.95 & 0.438 \\
50 & 0.802 & 0.662 & 0.300 \\
40 & 0.249 & 0.244 & 0.175 \\
30 & 0.101 & 0.100 & 0.0921 \\
20 & 0.0494 & 0.0490 & 0.0481 \\
10 & 0.0276 & 0.0274 & 0.0270 \\
0 & 0.0126 & 0.0126 & 0.0126 \\
\hline $\mathrm{T}$ & 100 & 10 & 1 \\
\hline
\end{tabular}

Cumulative

/Contributions af ionization events from various spectral regions of the energy degradation spectrum. For example, for an electron with initial energy $T_{0}=100 \mathrm{kEV}, 50$ percent of the ionization is produced by electrons (primary and secondaries) with energies less than $0.802 \mathrm{keV}$. 
T, keV dE/dx, $\mathrm{MeV} / \mathrm{g} \mathrm{cm}^{-2} \mathrm{~T}, \mathrm{keV} \mathrm{dE} / \mathrm{dx}, \mathrm{MeV} / \mathrm{g} \mathrm{cm}^{-2}$
(a)
(b)
(a)
(b)

\begin{tabular}{rrrlll}
\hline 100 & 4.14 & 4.14 & 0.3 & 200. & 230. \\
50 & 6.65 & 6.65 & 0.2 & 221. & 257. \\
30 & 9.73 & 9.73 & 0.1 & 221. & \\
20 & 13.3 & 13.3 & 0.05 & 130. & \\
10 & 22.5 & 22.8 & 0.03 & 67.7 & \\
5 & 36.3 & 38.8 & 0.02 & 27.8 & \\
3 & 53.5 & 56.9 & 0.01 & 2.86 & \\
2 & 70.4 & 76.2 & 0.005 & 0.035 & \\
1 & 110. & 122. & 0.003 & 0.0021 & \\
0.5 & 165. & 183. & 0.002 & 0.013 & \\
\hline
\end{tabular}

Stopping Power for Electrons in Water Vapor

(a) Present work; derived from detailed cross sections for ionization and excitation.

(b) From Bethe stopping-power formula, evaluated with mean excitation energy $I=71.7 \mathrm{eV}$. 
TABLE 9

\begin{tabular}{|c|c|c|c|}
\hline $\begin{array}{c}\text { ELECTRON } \\
\text { ENERGY } \\
(\mathrm{eV})\end{array}$ & $\begin{array}{c}\text { CSDA } \\
\text { RANGE } \\
\left(\mathrm{g} \mathrm{cm} \mathrm{cm}^{-2}\right)\end{array}$ & $\begin{array}{c}\text { ELECTRON } \\
\text { ENERGY } \\
(\mathrm{eV})\end{array}$ & $\begin{array}{c}\text { CSDA } \\
\text { RANGE } \\
\left(\mathrm{g} \mathrm{cm}^{-2}\right)\end{array}$ \\
\hline 100,000 & $1.432 \times 10^{-2}$ & 1000 & $7.156 \times 10^{-6}$ \\
\hline 80,000 & $9.723 \times 10^{-3}$ & 800 & $5.446 \times 10^{-6}$ \\
\hline 60,000 & $5.913 \times 10^{-3}$ & 600 & $3.980 \times 10^{-6}$ \\
\hline 50,000 & $4.303 \times 10^{-3}$ & 500 & $3.343 \times 10^{-6}$ \\
\hline 40,000 & $2.911 \times 10^{-3}$ & 400 & $2.764 \times 10^{-6}$ \\
\hline 30,000 & $1.756 \times 10^{-3}$ & 300 & $2.236 \times 10^{-6}$ \\
\hline 20,000 & $8.635 \times 10^{-4}$ & 200 & $1.761 \times 10^{-6}$ \\
\hline 15,000 & $5.234 \times 10^{-4}$ & 150 & $1.540 \times 10^{-6}$ \\
\hline 10,000 & $2.828 \times 10^{-4}$ & 100 & $1.319 \times 10^{-6}$ \\
\hline 8000 & $1.799 \times 10^{-4}$ & 80 & $1.223 \times 10^{-6}$ \\
\hline 6000 & $1.096 \times 10^{-4}$ & 50 & $1.037 \times 10^{-6}$ \\
\hline 5000 & $8.095 \times 10^{-5}$ & 40 & $9.509 \times 10^{-7}$ \\
\hline 4000 & $5.615 \times 10^{-5}$ & 30 & $8.333 \times 10^{-7}$ \\
\hline 3000 & $3.538 \times 10^{-5}$ & 20 & $6.103 \times 10^{-7}$ \\
\hline 2000 & $1.890 \times 10^{-5}$ & 15 & $3.241 \times 10^{-7}$ \\
\hline 1500 & $1.240 \times 10^{-5}$ & 12.6 & 0.0 \\
\hline
\end{tabular}

Electron range in water vapor. 


\begin{tabular}{|c|c|c|c|c|c|c|}
\hline \multirow{2}{*}{$\begin{array}{l}\Delta \\
(\mathrm{eV})\end{array}$} & \multicolumn{3}{|c|}{$100 \mathrm{keV}$} & \multicolumn{3}{|c|}{$50 \mathrm{keV}$} \\
\hline & (a) & (b) & $\%$ DIFF & (a) & (b) & $\%$ DIFF \\
\hline 400 & 0.6940 & 0.6672 & 3.9 & 0.7167 & 0.6855 & 4.4 \\
\hline 200 & 0.6467 & 0.6001 & 7.2 & 0.6649 & 0.6160 & 7.3 \\
\hline 100 & 0.5993 & 0.5267 & 12.1 & 0.6128 & 0.5408 & 11.8 \\
\hline 50 & 0.5519 & 0.4551 & 17.5 & 0.5607 & 0.4674 & 16.6 \\
\hline
\end{tabular}

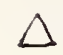

$(\mathrm{eV})$

400

200

100

50

$\Delta$

$(\mathrm{eV})$

400

200

100

50
$30 \mathrm{keV}$

(a) (b) $\%$ DIFF

0.7355

0.6982

5.1

0.6797

0.6265

7.8

0.6237

0.5499

11.8

0.5675

0.4750

16.3

$10 \mathrm{keV}$

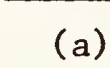

(b) \% DIFF

0.7854

0.7393

5.9

0.7191

0.6616

8.0

0.6518

0.5804

11.0

0.5840

0.5006
$20 \mathrm{keV}$

\begin{tabular}{ccr}
\hline (a) & (b) & DIFF \\
0.7522 & 0.7088 & 5.8 \\
0.6929 & 0.6352 & 8.3 \\
0.6331 & 0.5574 & 11.9 \\
0.5731 & 0.4812 & 16.0
\end{tabular}

$5 \mathrm{keV}$

(a)

(b) $\%$ DIFF

0.8262

0.7854

4.9

0.7518

0.7029

6.5

0.6752

0.6168

8.6

0.5974

0.5316

11.0

Ratio of the restricted to the total electron stopping power in water vapor, as a function of the cut-off energy $\Delta$.

(a) Calculated according to the Bethe theory

(b) Derived from the excitation and ionization cross sections used in the present work.

Note that the restricted stopping power excludes energy transfers in which the secondary electron acquires a kinetic energy greater than $\triangle$. 
NBS-114A (REV. 7-73)

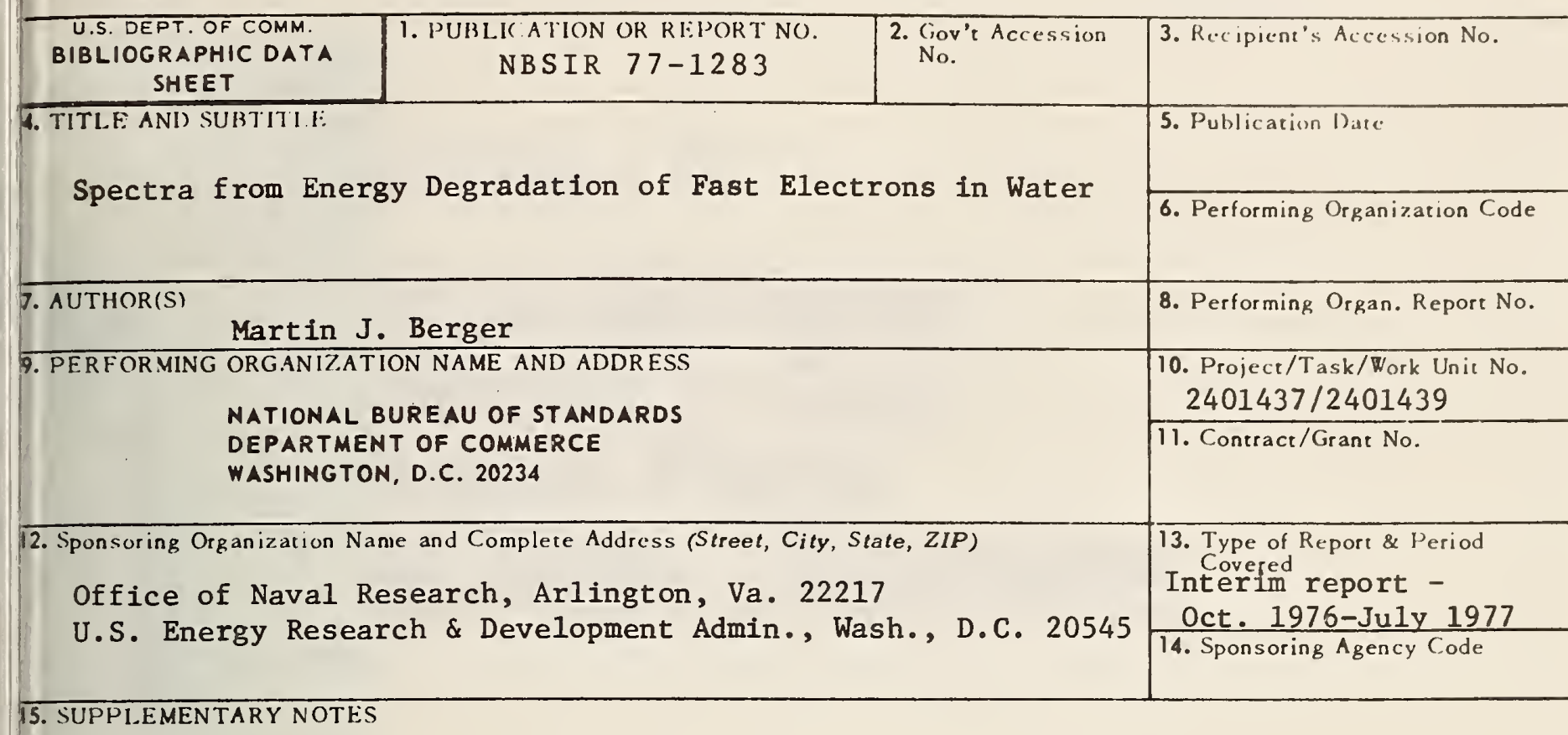

\section{SUPPILEENTARY NOTES}

16. ABSTRACT (A 200-word or less factual summary of most significant information. If document includes a significant bibliography or literature survey, mention it here.)

This report gives data for energy degradation spectra in water vapor. Calculations utilized the Monte Carlo method and included Auger electrons. Results include 1) a new energy scaling principle at low energies, 2) values difference source energies for $W$, the mean energy loss per ion pair formed, 3) data on the electron range calculated by continuous-slowing-down approximation, and 4) data on stopping powers and restricted stopping powers as compared with results from the Bethe theory.

7. KEY WORDS (six to twelve entries; alphabetical order; capitalize only the first letter of the first key word unless a proper name; separated by semicolons)

Charged particle transport; electron energy degradation; electron penetration; slowing-down spectra; stopping power in water; $W$-value in water.

\begin{tabular}{|c|c|c|}
\hline $\begin{array}{l}\text { 8. AVAILABILITY } \\
\text { X For Official Distribution. Do Not Release to NTIS }\end{array}$ & $\begin{array}{l}\text { 19. SECURITY CLASS } \\
\text { (THIS REPURT) } \\
\text { X } \\
\text { UNCL ASSIFIED }\end{array}$ & 21. NO. OF PAGES \\
\hline $\begin{array}{l}\text { L.] Order From Sup. of Doc., U.S. Government Printing Office } \\
\text { Washington, D.C.. 20402, SDCat. No.C13 }\end{array}$ & $\begin{array}{l}\text { 20. SECURITY CLASS } \\
\text { (THIS PAGE) }\end{array}$ & 22. Price \\
\hline $\begin{array}{l}{\left[\begin{array}{l}\text { Order From National Technical Information Service (NTIS) } \\
\text { Springfield, Virginia } 22151\end{array}\right.}\end{array}$ & UNCLASSIFIED & \\
\hline
\end{tabular}


